\title{
A continuum modeling approach to the spatial analysis of air quality and housing location choice
}

\author{
Jun Yin ${ }^{1}$, S.C. Wong ${ }^{1}$, Keechoo Choi ${ }^{2}$, Y.C. Du ${ }^{3}$ \\ ${ }^{1}$ Department of Civil Engineering, The University of Hong Kong, Pokfulam Road, \\ Hong Kong, China \\ ${ }^{2}$ Department of Transportation Engineering, TOD-based Sustainable Urban Transportation \\ Center, Ajou University, Republic of Korea \\ ${ }^{3}$ Key Lab. of Road and Traffic Engineering of the Ministry of Education, Tongji University, \\ Shanghai, China
}

\begin{abstract}
Today, air pollution is a great issue, and the transport sector is an important emission source. In this study, we present an integrated land use, transport and environment model in which transport-related pollutants are assumed to influence people's housing location choices, and a continuum modeling approach is applied. The pollutants generated by the transport sector are dispersed by the wind and they affect air quality. The air quality changes people's housing choices, which in turn changes their travel behavior. We assume that the road users are continuously distributed over the city, that the road network is relatively dense, and that this network can be approximated as a continuum. The total demand is categorized into several classes, and the modeled region contains several subdistricts. People who live in different subdistricts or who belong to different classes of commuters are assumed to have different perceptions of travel time, air quality and the housing provision-demand relationship. The finite element method and the Newton-Raphson algorithm are adopted to solve this problem, and a numerical valuation is given to illustrate the effectiveness and efficiency of the proposed model.
\end{abstract}

Key words: housing choice, air quality, continuum model, spatial analysis, urban city

\section{Introduction}

The housing location choice model is fundamental to land use models, and ultimately to travel demand models. The prediction of housing location choices is important for making decisions on how to allocate housing development and commercial activities.

Traditionally, the housing location choice has been viewed as a tradeoff between transport costs and housing prices (Giuliano, 1989). Wheaton (1977) found that when the elasticity of housing prices exceeds the travel costs, people may choose to live further from their work locations. Rosen (1974) formulated the theory of hedonic prices to explain the spatial equilibrium between provider and buyer. The idea of hedonic prices was later widely adopted in studies of housing prices (Huh and Kwak, 1997, Orford, 2000). However, Ellickson (1981) pointed out that hedonic prices cannot predict consumer behavior. Since that study, logit models have been used in studies to predict housing location choices (Ben-Akiva and Bowman, 1998, Bhat and Guo, 2004, Zongdag and Pieters, 2005). In these studies, various kinds of detailed information about houses and households are considered, including the sizes and neighboring environments of houses, the structures of families and the types or times of travel. Based on the interactions between land use and transport, discrete modeling techniques such as logit models have been used by several researchers to develop integrated 
land use, transport and environment models (Wegener and Fuerst, 2004, Wegener, 2004, Wagner and Wegener, 2007). These models consider how land use influences transport type, and how transport-related pollutants and noise affect land-use patterns.

In applying such models to a two-dimensional region, one factor to consider is that many transport-related decision variables are continuous, especially housing location choices and travel destinations. In this type of situation, discrete modeling techniques do not work properly. However, there is another type of logit model, known as a continuous logit model (Ben-Akiva et al., 1985, Ben-Akiva and Wantantada, 1981), which can be applied to consider continuous choice settings.

Ho and Wong $(2007,2005)$ incorporated a continuous logit model into a continuum modeling frame to study travel patterns and housing location choices in an urban city. They assumed that within the modeled region, all of the variables were continuous, and that the differences between adjacent areas were slight. Thus, the transportation system in the city could be described by smooth mathematical functions (Vaughan, 1987; Wong and Wong, 2015, 2016). Compared to the discrete modeling approach, the continuum modeling approach has several inherent advantages (Ho and Wong, 2006). First, rather than modeling the actual number of links/nodes as is done in the discrete modeling approach, the continuum modeling approach approximates the region as a continuum, and thus reduces the problem of size. Second, in the modeling setup process, the continuum approach requires less data, which makes this approach more suitable for an initial planning stage in which data availability is limited. Third, the continuum approach provides a better understanding of global characteristics such as travel demand, land development intensity and travel cost. Fourth, the results of the continuum approach are more realistic, because the discrete modeling approach normally uses only one node to represent one zone, and the OD matrix is the OD between these zones, even though the characteristics within a zone may differ greatly. Nevertheless, the continuum modeling approach can also work well with discrete modeling approach in a mixed discrete/continuum modeling framework (Du et al., 2016).

Due to the inherent advantages of the continuum modeling approach, this study generally follows the works of Ho and Wong (2005), Wong (1998) and Wong et al. (1998) in considering a continuum transportation system. It is assumed that there are no sources of emissions other than transport in the modeled region. In addition to transport costs and housing prices, the air quality is a third factor that influences people's choices of residence. Here, an integrated model of land use, transport and environment is introduced within the frame of the continuum modeling approach, and an attempt is made to solve the problems involved by applying the finite element method (FEM). A numerical example is given in the final section.

\section{Model formulation}

In this section, a city of arbitrary shape and with more than one central business district (CBD) is studied. The modeled region can be divided into several subdistricts, as shown in Figure 1. The road network outside the CBDs is assumed to be relatively dense, so that it can be approximated as a continuum. Transport demand and housing provision are continuously distributed around the CBDs, and all of the employment happens within the CBDs. People travel between their homes and the CBDs along the least costly routes during peak hours. Their choices of destination are determined by their perceptions of the travel costs and the externalities of the CBDs. People who live in different subdistricts or who belong to different 
user classes are assumed to have different perceptions of travel time, air quality and the housing provision-demand relationship. We denote the region of the city center as $\Omega$, the outer boundary of the city as $\Gamma$, the location of the CBD $n$ as $O_{n}$ and the boundary of the CBD $n$ as $\Gamma_{c n}$. We also assume that no road users will travel across the city's outer boundary $(\Gamma)$.

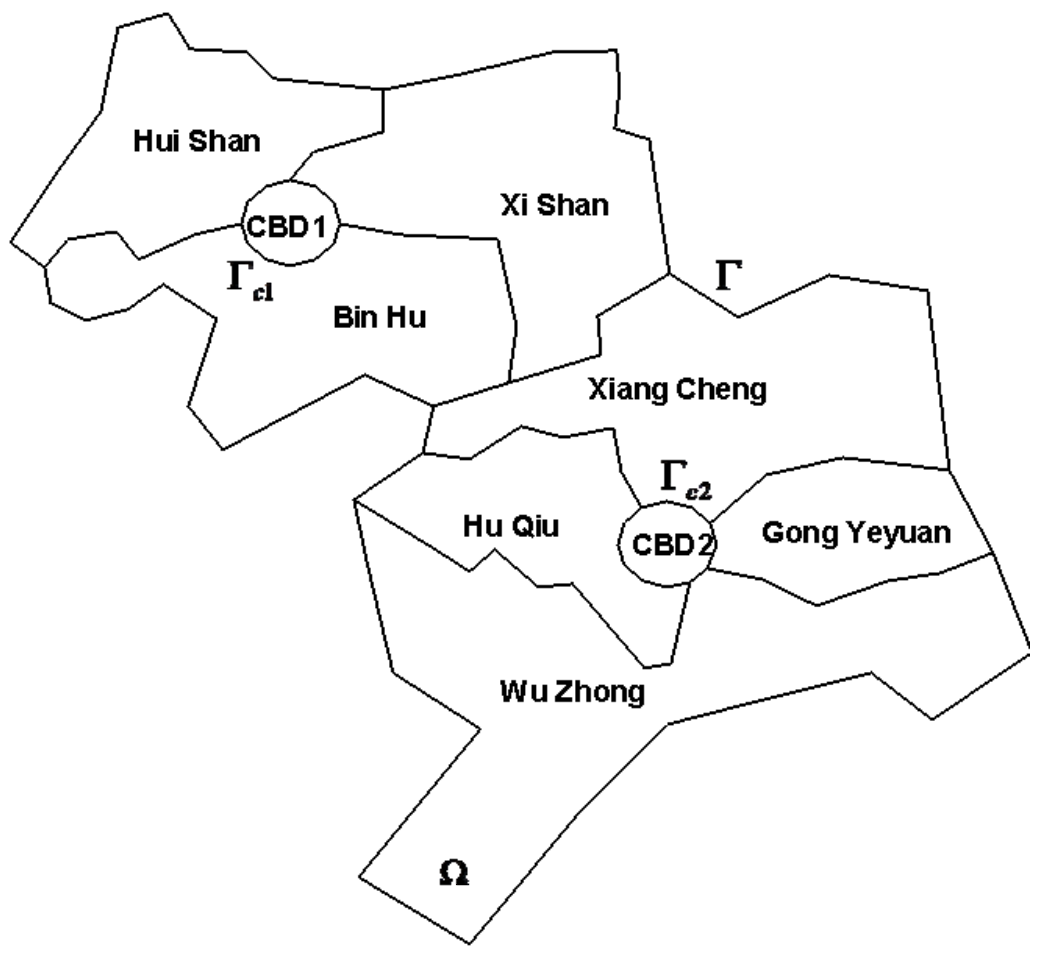

Figure 1: The modeled city

At location $(x, y)$, we define $\mathbf{f}_{m n}(x, y)=\left(f_{x m n}(x, y), f_{y m n}(x, y)\right)$ as the flow vector of class $m$ road users who travel to the CBD $n$ (expressed as the number of commuters who cross a unit width), where $f_{x m n}(x, y)$ and $f_{y m n}(x, y)$ are the flow flux in the $x$ and $y$ directions, respectively. $\left|\mathbf{f}_{m n}(x, y)\right|=\sqrt{f_{x m n}(x, y)^{2}+f_{y m n}(x, y)^{2}}$ is the corresponding flow intensity. At a particular location $(x, y)$ and for a given flow pattern $\mathbf{f}_{m n}(x, y)$, we define the traffic speed as a monotonic decreasing function of the total flow intensity, $F(x, y)=\sum_{n=1}^{N_{n}} \sum_{m=1}^{N_{m}}\left|\mathbf{f}_{m n}(x, y)\right|$, i.e., $v(x, y)=v(F(x, y), x, y)$. To take the relationship between acceleration and speed into consideration, the following equations must be satisfied.

$$
\begin{aligned}
& a_{x m n}(x, y)-v_{x m n}(x, y) \frac{\partial v_{x m n}(x, y)}{\partial x}=0, \forall(x, y) \in \Omega, m \in N_{m}, n \in N_{n} \\
& a_{y m n}(x, y)-v_{y m n}(x, y) \frac{\partial v_{y m n}(x, y)}{\partial y}=0, \forall(x, y) \in \Omega, m \in N_{m}, n \in N_{n}
\end{aligned}
$$

where $\mathbf{a}_{m n}(x, y)=\left(a_{x m n}(x, y), a_{y m n}(x, y)\right)$ is the acceleration vector of class $m$ commuters heading to the $\operatorname{CBD} n ; a_{x m n}$ and $a_{y m n}$ represent acceleration in the $x$-direction and $y$ - 
direction, respectively; $\mathbf{v}_{m n}(x, y)=\left(v_{x m n}(x, y), v_{y m n}(x, y)\right)$ is the velocity vector of class $m$ commuters heading to the $\operatorname{CBD} \quad n ; \quad v_{x m n}(x, y)=v(x, y) \frac{f_{x m n}(x, y)}{\left|\mathbf{f}_{m n}(x, y)\right|} \quad$ and $v_{y m n}(x, y)=v(x, y) \frac{f_{y m n}(x, y)}{\left|\mathbf{f}_{m n}(x, y)\right|}$ are the velocity in the $x$-direction and $y$-direction (respectively) for class $m$ commuters to patronize CBD $n ; N_{m}$ is the total number of user classes, and $N_{n}$ is the total number of CBDs.

We next define the travel cost potential. Let $c(x, y)$ be the local travel cost, which is related to the travel speed as

$$
c(x, y)=1 / v(x, y)
$$

where the travel cost is expressed in hours per unit length of movement at location $(x, y)$. As travel speed is a monotonic decreasing function of the total flow intensity, we define a BPRtype relationship between local travel cost and traffic intensity as follows:

$$
c(x, y)=1 / v(x, y)=c_{0}(x, y)+\eta(x, y)\left(\sum_{n=1}^{N_{n}} \sum_{m=1}^{N_{m}}\left|\mathbf{f}_{m n}(x, y)\right|\right)^{\kappa(x, y)}, \forall(x, y) \in \Omega
$$

where $c_{0}(x, y)$ is the free-flow travel time, $\eta(x, y)$ and $\kappa(x, y)$ are the congestion sensitivity parameters at location $(x, y)$. Let $p_{m}$ be the value of time for class $m$ commuters. The travel cost of class $m$ commuters can be expressed in CNY per unit length of travel at $(x, y)$ as

$$
c_{m}(x, y)=p_{m} c(x, y), \forall(x, y) \in \Omega, m \in N_{m}
$$

For a given flow pattern $\mathbf{f}_{m n}(x, y)$ and a unit travel cost $c_{m}(x, y)$, we consider the function $u_{m n}(x, y)$, which is the transportation cost of class $m$ commuters at location $(x, y)$ in traveling to the $\operatorname{CBD} n$. The following equation should be satisfied:

$$
c_{m}(x, y) \frac{\mathbf{f}_{m n}(x, y)}{\left|\mathbf{f}_{m n}(x, y)\right|}+\nabla u_{m n}(x, y)=0, \forall(x, y) \in \Omega, m \in N_{m}, n \in N_{n}
$$

Also, equation (6) is equivalent to the following equations (7) and (8):

$$
\begin{aligned}
& c_{m}(x, y) \frac{f_{x m n}(x, y)}{\left|\mathbf{f}_{m n}(x, y)\right|}+\frac{\partial u_{m n}(x, y)}{\partial x}=0, \forall(x, y) \in \Omega, m \in N_{m}, n \in N_{n} \\
& c_{m}(x, y) \frac{f_{y m n}(x, y)}{\left|\mathbf{f}_{m n}(x, y)\right|}+\frac{\partial u_{m n}(x, y)}{\partial y}=0, \forall(x, y) \in \Omega, m \in N_{m}, n \in N_{n}
\end{aligned}
$$

In equation (6), the flow vector is the direct opposite of the gradient of the scalar function $u_{m n}(x, y)$, that is,

$$
-\mathbf{f}_{m n}(x, y) / / \nabla u_{m n}(x, y), \forall \mathbf{f}_{m n} \neq 0,(x, y) \in \Omega
$$

where "//" means that the two vectors are traveling in the same direction.

For each type of commuters, the flow vector and trip demand must satisfy the flow conservation conditions in their region of the city.

$$
\nabla \mathbf{f}_{m n}(x, y)-q_{m n}(x, y)=0, \forall(x, y) \in \Omega, m \in N_{m}, n \in N_{n}
$$


where $\nabla \mathbf{f}_{m n}(x, y)=\frac{\partial f_{x m n}}{\partial x}+\frac{\partial f_{y m n}}{\partial y}$ is the gradient of the flow vector $\mathbf{f}_{m n}(x, y)$, and $q_{m n}(x, y)$ is the travel demand of the class $m$ commuters at location $(x, y)$ who will travel to the CBD $n$. This density is expressed as the number of commuters per unit area per unit of time.

Apart from the transportation cost, there is also a cost incurred in each CBD, which is known as the market externality. This externality is specified as a function of the market share of the facility, which is represented as $S_{m n}$ (expressed in the same unit as the transportation cost, namely CNY) for the class $m$ commuters' perception of travel cost to the CBD $n$. This externality could have a decreasing value, to represent a positive externality (in economies of scale or of employment opportunities), or it could have an increasing value for a negative externality (i.e., congestion). The value could also be convex in a case with a positive externality when the share is small, and a negative externality when the share is large. This convex pattern may arise because some cost is fixed (such as the investment in the infrastructure) for the CBD, as such fixed costs should be shared by all of the road users. In that case, the larger the volume of demand, the smaller the cost of this factor. However, more demand will also cause severe congestion, which can result in more delay and increased cost. In our model, $S_{m n}$ is defined as a function of $V_{n}$ (the total demand for the CBD $n$ ), $V_{n}=\sum_{m=1}^{N_{m}} \iint_{\Omega} q_{m n} d \Omega$, and $V_{n}$ is sum of all classes of road users who will travel to the CBD $n$. Then, the perceived cost of class $m$ users traveling to the CBD $n$ can be denoted as $C_{m n}$

$$
C_{m n}=\theta_{m n}+S_{m n}\left(V_{n}\right)
$$

where $\theta_{m n}$ is the biased component that represents the preference of class $m$ users traveling to the CBD $n$. The total perceived cost for each class of road users to the CBDs can then be defined as $\Upsilon_{m n}$, where

$$
\Upsilon_{m n}(x, y)=C_{m n}+u_{m n}(x, y), \forall(x, y) \in \Omega, m \in N_{m}, n \in N_{n}
$$

For any route $p$ used by class $m$ commuters from location $(H)$ to the CBD $n$, the total perceived cost (including the transportation cost and the CBD's externalities) is

$$
\begin{aligned}
C_{p} & =C_{m n}+\int_{p} C_{m} d s=C_{m n}+\int_{p} C_{m} \frac{\mathbf{f}_{m n}}{\left|\mathbf{f}_{m n}\right|} \mathbf{d s}=C_{m n}-\int_{p} \nabla u_{m n} \mathbf{d s}=C_{m n}-\left(u_{m n}\left(O_{n}\right)-u_{m n}(H)\right) \\
& =C_{m n}+u_{m n}(H)
\end{aligned}
$$

where $u_{m n}\left(O_{n}\right)=0$ at $\operatorname{CBD} n, O_{n}$. For any route $\bar{p}$ used by class $m$ travelers from location $(H)$ to the CBD $n$, the total perceived cost (including the transportation cost and the CBD's externalities) is

$$
\begin{aligned}
C_{\bar{p}} & =C_{m n}+\int_{\bar{p}} C_{m} d s \geq C_{m n}+\int_{\bar{p}} C_{m} \frac{\mathbf{f}_{m n}}{\left|\mathbf{f}_{m n}\right|} \mathbf{d s}=C_{m n}-\int_{\bar{p}} \nabla u_{m n} \mathbf{d s}=C_{m n}-\left(u_{m n}\left(O_{n}\right)-u_{m n}(H)\right) \\
& =C_{m n}+u_{m n}(H)=C_{p}
\end{aligned}
$$

The inequality in equation (14) results from the fact that for route $\bar{p}$, the vectors $\mathbf{f}_{m n} /\left|\mathbf{f}_{m n}\right|$ and $\mathbf{d s}$ are not parallel, and in that case $d s \geq\left(\mathbf{f}_{m n} /\left|\mathbf{f}_{m n}\right|\right) \cdot \mathbf{d s}$. So, for the unused routes, the total perceived cost is at least equal to that of the used routes. This model thus guarantees that the users will choose the least costly route between their destinations and their homes in a user-optimal manner. 
For a particular class of users, the probability of a user choosing a CBD as his destination depends on the total perceived cost from his home to his destination, which is governed by a logit-type distribution:

$$
q_{m n}(x, y)=q_{m}(x, y) \frac{\exp \left(-\chi_{m} \Upsilon_{m n}(x, y)\right)}{\sum_{i=1}^{N_{n}} \exp \left(-\chi_{m} \Upsilon_{m i}(x, y)\right)}, \forall(x, y) \in \Omega, m \in N_{m}, n \in N_{n}
$$

where $q_{m}(x, y)=\sum_{i=1}^{N_{n}} q_{m i}(x, y)$ is the total travel demand at location $(x, y)$, and $\chi_{m}$ is a sensitivity parameter, of class $m$ commuters. Then we define $\Pi_{m}(x, y)$ as a function of the log-sum cost of the users traveling from the location $(x, y)$ to all of the CBDs.

$$
\Pi_{m}(x, y)=-\frac{1}{\chi_{m}} \ln \sum_{i=1}^{N_{n}} \exp \left(-\chi_{m} \Upsilon_{m i}(x, y)\right), \forall(x, y) \in \Omega, m \in N_{m}
$$

We now define the utility function for class $m$ commuters, $U_{m}(x, y)=\Pi_{m}(x, y)+\tau_{m}(x, y)+r_{m}(x, y)$, which consists of three components. $\Pi_{m}(x, y)$ is the log-sum cost, as obtained from equation (16). $\tau_{m}(x, y)$ is class $m$ commuters' perception of the air quality, which is linear to the local pollutant concentration $\tau_{m}(x, y)=\zeta_{m} t(x, y)$, where $\zeta_{m}$ is a parameter that measures the sensitivity of class $m$ commuters to air quality, and $t(x, y)$ is the pollutant concentration at location $(x, y)$. The housing rent $r_{m}(x, y)$ depends on the total demand density $q(x, y)=\sum_{m=1}^{N_{m}} q_{m}(x, y)$ and the total housing supply density $H(x, y)$, which are estimated as follows:

$$
r_{m}(x, y)=\alpha_{m}(x, y)(1+\beta(x, y) q(x, y) /(H(x, y)-q(x, y)))
$$

where $\alpha_{m}(x, y)$ represents the different commuters' perceptions of the housing rents, $\beta(x, y)$ are scalar parameters that represent the demand-dependent components of the rent function at location $(x, y)$, and $H(x, y) \geq q(x, y)$.

The interaction between housing allocation and traffic equilibrium is governed by the demand distribution function, which is used to describe the way in which road users choose their home locations in the city. Ho and Wong (2005) identified housing rent and travel cost as the basic variables that affect commuters' choices of where to live. In the case examined in this study, the externalities of the CBDs and the local air quality are also considered. The following equation is used to incorporate the housing allocation problem into the transportation equilibrium problem.

$$
q_{m}(x, y)-Q_{m} \frac{\exp \left(-\gamma_{m} U_{m}(x, y)\right)}{\iint_{\Omega} \exp \left(-\gamma_{m} U_{m}(x, y)\right) d \Omega}=0, \forall(x, y) \in \Omega, m \in N_{m}
$$

where $Q_{m}$ is the total demand of class $m$ commuters, which is fixed in this model; $U_{m}$ is the utility function perceived by class $m$ commuters to all of the CBDs from the location $(x, y)$, and $\gamma_{m}$ is a positive scalar parameter that measures the model's sensitivity.

Combining equations (15) and (18) reveals that for $\forall(x, y) \in \Omega, m \in N_{m}, n \in N_{N}$, 


$$
q_{m n}(x, y)-Q_{m} \frac{\exp \left(-\gamma_{m} U_{m}(x, y)\right)}{\iint_{\Omega} \exp \left(-\gamma_{m} U_{m}(x, y)\right) d \Omega} \times \frac{\exp \left(-\chi_{m} \Upsilon_{m n}(x, y)\right)}{\sum_{i=1}^{N_{n}} \exp \left(-\chi_{m} \Upsilon_{m i}(x, y)\right)}=0
$$

We also consider the boundary conditions that must be satisfied.

$$
\begin{gathered}
u_{m n}=0, \forall(x, y) \in \Gamma_{c n}, \forall m \in N_{m}, n \in N_{n} \\
\mathbf{f}_{m n}=0, \forall(x, y) \in \Gamma, \Gamma_{c k}, \forall m \in N_{m}, n, k \in N_{n}, n \neq k
\end{gathered}
$$

In equation (20), as the users at $\Gamma_{c n}$ are already at the boundary of the CBD $n$, they will incur no transportation cost in traveling to the CBD $n$. In equation (21), we assume that there is no traffic flow across the city boundary, and that class $m$ road users do not enter any CBD other than the CBD they are heading toward.

In this model, it is assumed that the transportation sector is the only source of emissions. The pollutants generated from moving vehicles will disperse in a certain manner, which will then affect the air quality. The concentration of pollutants influences people's decisions about their housing locations. There are several models for estimating transport-related pollutant emissions. Here, the acceleration and speed-based model is used, in which the emission rate is defined as a function of vehicle type, instantaneous speed and acceleration. In this study, we apply the model proposed by Ahn et al. (2002, 1999), in which the emission rate is defined as a function of the instantaneous acceleration and speed, as follows:

$$
E^{k}=\exp \left(\sum_{i=0}^{3} \sum_{j=0}^{3} \omega_{i, j}^{k} v^{i} a^{j}\right)
$$

where $a$ is the instantaneous acceleration $\left(\mathrm{km} / \mathrm{h}^{2}\right) ; v$ is the instantaneous speed $(\mathrm{km} / \mathrm{h}) ; \omega_{i, j}^{k}$ is the model regression coefficient for speed power $i$ and acceleration power $j$, and $E^{k}$ is the instantaneous fuel consumption and emissions rate with the superscript $(k)$ denoting different kinds of emissions $\mathrm{HC}, \mathrm{CO}, \mathrm{NO}_{x}(\mathrm{mg} / \mathrm{s})$, and fuel consumption $\mathrm{FC}$ (gal/h).

There is no single representative measure of traffic related air pollution. However, as suggested by Wardman and Bristow (2004), $\mathrm{NO}_{x}$ levels can be usefully selected, as this pollutant has clearly adverse health effects. Here, we consider only the $\mathrm{NO}_{x}$ emissions. The parameters involved are summarized in the following Table 1.

Table 1: Parameters for estimating $\mathrm{NO}_{x}$ emissions

\begin{tabular}{ccccccc}
\hline$i, j$ & 0,0 & 0,1 & 0,2 & 0,3 & 1,0 & 2,0 \\
\hline$\omega_{i, j}^{\mathrm{NO}_{x}}$ & $-1.07 \mathrm{E}+00$ & $1.00 \mathrm{E}+03$ & $1.38 \mathrm{E}+05$ & $-5.86 \mathrm{E}+07$ & $5.09 \mathrm{E}-02$ & $-2.05 \mathrm{E}-04$ \\
\hline$i, j$ & 3,0 & 1,1 & 2,1 & 3,1 & 1,2 & \\
\hline$\omega_{i, j}^{\mathrm{NO}_{x}}$ & $7.52 \mathrm{E}-07$ & $6.71 \mathrm{E}+01$ & $-6.18 \mathrm{E}-01$ & $1.71 \mathrm{E}-03$ & $-4.92 \mathrm{E}+04$ & \\
\hline$i, j$ & 2,2 & 3,2 & 1,3 & 2,3 & 3,3 & \\
\hline$\omega_{i, j}^{\mathrm{NO}_{x}}$ & $-1.11 \mathrm{E}+03$ & $4.95 \mathrm{E}+00$ & $-2.16 \mathrm{E}+07$ & $-1.44 \mathrm{E}+05$ & $-1.06 \mathrm{E}+03$ & \\
\hline
\end{tabular}

Under the route choice governed by user-optimal conditions, commuters may accelerate or decelerate along their trajectory, according to the spatial variation in traffic conditions in the neighboring area. The acceleration in the direction of movement is determined by

$$
a=\left(a_{x} f_{x}+a_{y} f_{y}\right) / \sqrt{f_{x}^{2}+f_{y}^{2}} .
$$


In the continuum model, vehicles are spread across the city continuously, and can be viewed as an area-based emissions source that represents an integration of the point-based emissions sources. The emissions rate from each point is constant in the static continuum model, so the basic Gaussian dispersion model can be directly applied. In Gaussian dispersion model (EPA, 2010, 2011),

$$
\bar{t}(x, y, z, h)=\frac{Q}{2 \pi u \sigma_{y} \sigma_{z}} \exp \left(-\frac{y^{2}}{2 \sigma_{y}{ }^{2}}\right)\left\{\exp \left[-\frac{(z-h)^{2}}{2 \sigma_{z}^{2}}\right]+\exp \left[-\frac{(z+h)^{2}}{2 \sigma_{z}{ }^{2}}\right]\right\}
$$

where $\bar{t}$ is defined as the pollutant concentration for a single-point emissions source that has an emission rate of $Q, x$ is the distance that the pollutants travel in the downwind direction, $y$ is the cross-wind distance from the pollution source measured along the perpendicular direction to the wind direction, $z$ is the height at which the pollution concentration is concerned, $h$ is the height of the emissions source, $u$ is the wind speed along the same direction as $x$, and $\sigma_{y}=\mu_{1} x\left(1+\rho_{1} x\right)^{-0.5}, \sigma_{z}=\mu_{2} x\left(1+\rho_{2} x\right)^{-0.5}$ are the parameters determined by the Pasquill stability, which is the combined effect of the weather conditions and the topographical roughness of the city, and this factor represents the speed at which pollutants disperse. It is assumed that no chemical transformations occur during dispersal. A typical dispersion type with an emissions source at point $(0,0,0)$ is plotted in Figure 2 . It is assumed that the wind direction is the same as $x$, and that the pollutant concentration is measured at ground level $(z=0, h=0)$.

As this model considers the concentration of emissions at ground level, $z=0$, the pollutant concentration at point $\left(x_{p}, y_{p}\right)$ is an integration of all of the upwind point emissions sources,

$$
t\left(x_{p}, y_{p}\right)=\iint_{\substack{-\infty<x<x_{p} \\-\infty<y<\infty}} \frac{Q(x, y)}{\pi u \sigma_{y^{\prime}} \sigma_{z^{\prime}}} \exp \left(-\frac{\left(y_{p}-y\right)^{2}}{2 \sigma_{y^{\prime}}{ }^{2}}-\frac{h^{2}}{2 \sigma_{z^{\prime}}{ }^{2}}\right) d x d y
$$

where $t\left(x_{p}, y_{p}\right)$ is the pollution concentration at the ground level of location $\left(x_{p}, y_{p}\right)$, $\sigma_{y^{\prime}}=\mu_{1}\left(x_{p}-x\right)\left(1+\rho_{1}\left(x_{p}-x\right)\right)^{-0.5} \quad$ and $\quad \sigma_{z^{\prime}}=\mu_{2}\left(x_{p}-x\right)\left(1+\rho_{2}\left(x_{p}-x\right)\right)^{-0.5} \quad, \quad$ and $Q(x, y)=0, \forall(x, y) \notin \Omega$. 


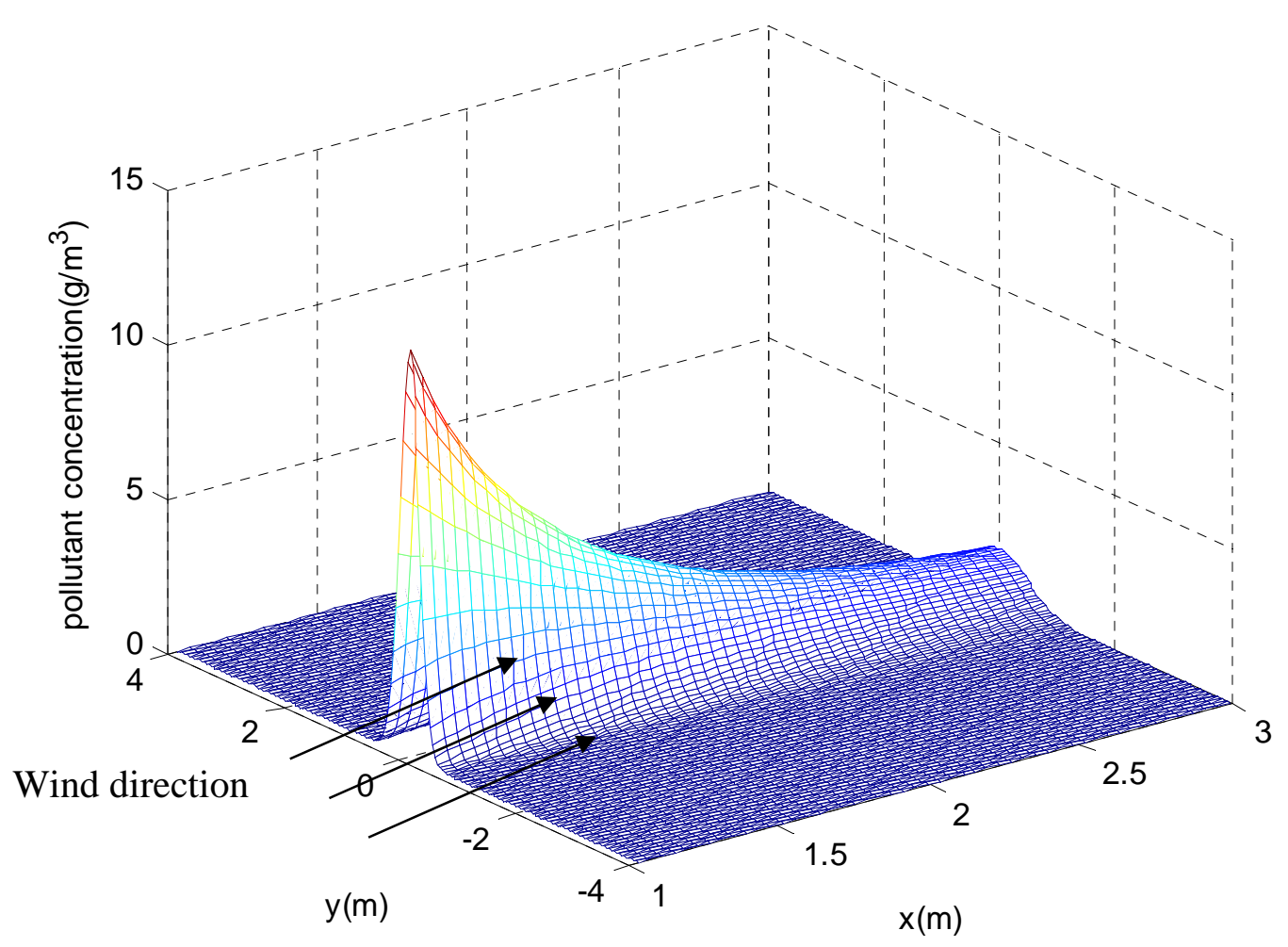

Figure 2: Gaussian dispersion type

\section{Solution algorithm}

The problem can be written as a system of differential equations, including equations (1), (2), (7), (8), (10) and (19), which can be solved by applying the finite element method (FEM). We first apply the weighted residual technique to transform these equations into the following expressions, where $w(x, y)$ is the weight function in the weighted residual technique, and this function can take any value. For $\forall(x, y) \in \Omega, m \in N_{m}, n \in N_{n}$, the following equations can be obtained:

$$
\begin{array}{r}
\iint_{\Omega}\left(a_{x m n}(x, y)-v_{x m n}(x, y) \frac{\partial v_{x m n}(x, y)}{\partial x}\right) w(x, y) d \Omega=0 \\
\iint_{\Omega}\left(a_{y m n}(x, y)-v_{y m n}(x, y) \frac{\partial v_{y m n}(x, y)}{\partial y}\right) w(x, y) d \Omega=0 \\
\iint_{\Omega}\left(c_{m}(x, y) \frac{f_{x m n}(x, y)}{\left|\mathbf{f}_{m n}(x, y)\right|}+\frac{\partial u_{m n}(x, y)}{\partial x}\right) w(x, y) d \Omega=0 \\
\iint_{\Omega}\left(c_{m n}(x, y) \frac{f_{y m n}(x, y)}{\left|\mathbf{f}_{m n}(x, y)\right|}+\frac{\partial u_{m n}(x, y)}{\partial y}\right) w(x, y) d \Omega=0 \\
\iint_{\Omega}\left(\nabla \mathbf{f}_{m n}(x, y)-q_{m n}(x, y)\right) w(x, y) d \Omega=0 \\
\iint_{\Omega}\left(q_{m n}(x, y)-Q_{m} \frac{\exp \left(-\gamma_{m} U_{m}(x, y)\right)}{\iint_{\Omega} \exp \left(-\gamma_{m} U_{m}(x, y)\right) d \Omega} \times \frac{\exp \left(-\chi_{m} \Upsilon_{m n}(x, y)\right)}{\sum_{i=1}^{N_{n}} \exp \left(-\chi_{m} \Upsilon_{m i}(x, y)\right)}\right) w(x, y) d \Omega=0
\end{array}
$$


After the region is discretized, we can set the local interpolation function $N(x, y)$ to $w(x, y)$, i.e., the Galerkin formulation. For a specific node $s$, the governing equations for all classes of commuters are given as follows.

$$
\mathbf{r}_{s m n}(\boldsymbol{\Psi})=\left\{\begin{array}{c}
\sum_{e \in T_{s}} \iint_{\Omega_{e}}\left(a_{x m n}(x, y)-v_{x m n}(x, y) \frac{\partial v_{x m n}(x, y)}{\partial x}\right) N_{s}(x, y) d \Omega \\
\sum_{e \in T_{s}} \iint_{\Omega_{e}}\left(a_{y m n}(x, y)-v_{y m n}(x, y) \frac{\partial v_{y m n}(x, y)}{\partial y}\right) N_{s}(x, y) d \Omega \\
\sum_{e \in T_{s}} \iint_{\Omega_{e}}\left(c_{m}(x, y) \frac{f_{x m n}(x, y)}{\left|\mathbf{f}_{m n}(x, y)\right|}+\frac{\partial u_{m n}(x, y)}{\partial x}\right) N_{s}(x, y) d \Omega \\
\sum_{e \in T_{s}} \iint_{\Omega_{e}}\left(c_{m}(x, y) \frac{f_{y m n}(x, y)}{\left|\mathbf{f}_{m n}(x, y)\right|}+\frac{\partial u_{m n}(x, y)}{\partial y}\right) N_{s}(x, y) d \Omega \\
\sum_{e \in T_{s}} \iint_{\Omega_{e}}\left(\nabla \mathbf{f}_{m n}(x, y)-q_{m n}(x, y)\right) N_{s}(x, y) d \Omega \\
\sum_{e \in T_{s}} \iint_{\Omega_{e}}\left(q_{m n}(x, y)-\frac{Q_{m} \exp \left(-\gamma_{m} U_{m}(x, y)\right)}{\iint_{\Omega} \exp \left(-\gamma_{m} U_{m}(x, y)\right) d \Omega} \times \frac{\exp \left(-\chi_{m} \Upsilon_{m n}(x, y)\right)}{\sum_{i=1}^{N_{n}} \exp \left(-\chi_{m} \Upsilon_{m i}(x, y)\right)}\right) N_{s}(x, y) d \Omega
\end{array}\right\}
$$

where $\Omega_{e}$ denotes the region of the elements $e ; T_{s}$ is the set of elements that connects with node $s ; N_{s}(x, y)$ is the local interpolation function of the element that connects with node $s$, $\boldsymbol{\Psi}$ is the solution vector, and $\mathbf{r}_{\text {smn }}$ is the nodal residual vector for class $m$ commuters who have a preference for CBD $n$ at node $s . \mathbf{r}_{s m n}=0$ means that the governing equations, (1), (2), (7), (8), (10) and (19), can be satisfied locally. For more details about the finite element method for continuum modeling, readers are referred to Wong et al. (1998).

For the global satisfaction of the governing equations, we require that

$$
\mathbf{R}(\boldsymbol{\Psi})=\operatorname{Col}\left(\mathbf{r}_{\text {smn }}(\boldsymbol{\Psi})\right)=0 \text {. }
$$

Boundary conditions (20) and (21) can be satisfied by forcing the specific variables to take a known value, as is very common in the FEM. For this system of non-linear equations, we can apply the Newton-Raphson algorithm with a line search to find a solution, for which we derive the iterative equation as follows:

$$
\boldsymbol{\Psi}_{k+1}=\boldsymbol{\Psi}_{k}-\lambda \mathbf{J}_{k}^{-1} \mathbf{R}_{k},
$$

where $\mathbf{J}_{k}$ is the Jacobian matrix of vector $\mathbf{R}_{k}$ at iteration $k$, and $\lambda$ is the step size, which is obtained by a line search to achieve the minimum $|\mathbf{R}(\boldsymbol{\Psi})|$. We compare the relative error $|\mathbf{R}(\boldsymbol{\Psi})| /|\boldsymbol{\Psi}|$ with the acceptable threshold $\varepsilon$. If $|\mathbf{R}(\boldsymbol{\Psi})| /|\boldsymbol{\Psi}|<\varepsilon$, then it is assumed that the solution to the system of equations (33) is found. The solution procedure is summarized as follows:

\section{Solution Procedure}

Step 1: Find an initial solution for $\boldsymbol{\Psi}_{0}$. Set $k=0$.

Step 2: Evaluate $\mathbf{R}\left(\boldsymbol{\Psi}_{k}\right)$ and $\mathbf{J}\left(\boldsymbol{\Psi}_{k}\right)$.

Step 3: If the relative error $\left|\mathbf{R}\left(\boldsymbol{\Psi}_{k}\right)\right| /\left|\boldsymbol{\Psi}_{k}\right|$ is less than the accepted error $\varepsilon$, then stop and take $\boldsymbol{\Psi}_{k}$ as the solution. 
Step 4: Otherwise, apply the line search method (with the smallest interval of $\delta$ ) to determine the step size $\lambda^{*}$ that minimizes the norm of the residual vector $\left|\mathbf{R}\left(\boldsymbol{\Psi}_{k+1}-\lambda \mathbf{J}_{k}^{-1} \mathbf{R}_{k}\right)\right|$.

Then, $\boldsymbol{\Psi}_{k+1}=\boldsymbol{\Psi}_{k}-\lambda^{*} \mathbf{J}_{k}^{-1} \mathbf{R}_{k}$.

Step 5: Replace $\boldsymbol{\Psi}_{k}$ with $\boldsymbol{\Psi}_{k+1}$. Set $k=k+1$, and go to Step 2 .

\section{Numerical example}

As an example, this study considers two neighboring cities in Jiangsu Province, Wuxi and Suzhou. It is assumed that people engage in activities in two places: their home and a CBD. However, only their activities in the CBDs generate a transportation demand. The parameters used are given in Table 2. Road users are classified into two classes. The total demand at the peak hours is assumed to be 400,000 units/h, and the proportion of Class 1 commuters is about $80 \%$, which amounts to 320,000 units/h.

Table 2: Parameters for different subdistricts

\begin{tabular}{cccccccc}
\hline \multirow{2}{*}{ Parameters } & $\begin{array}{c}\text { Bin } \\
\text { Hu }\end{array}$ & $\begin{array}{c}\text { Hui } \\
\text { Shan }\end{array}$ & $\begin{array}{c}\text { Xi } \\
\text { Shan }\end{array}$ & $\begin{array}{c}\text { Wu } \\
\text { Zhong }\end{array}$ & $\begin{array}{c}\text { Xiang } \\
\text { Cheng }\end{array}$ & $\begin{array}{c}\text { Hu } \\
\text { Qiu }\end{array}$ & $\begin{array}{c}\text { Gong Ye } \\
\text { Yuan }\end{array}$ \\
\hline$\gamma_{1}$ & 0.0016 & 0.0015 & 0.0015 & 0.0016 & 0.0015 & 0.0015 & 0.0016 \\
$\gamma_{2}$ & 0.0020 & 0.0021 & 0.0020 & 0.0021 & 0.0020 & 0.0021 & 0.0020 \\
$\chi_{1}$ & 0.014 & 0.012 & 0.012 & 0.014 & 0.012 & 0.012 & 0.014 \\
$\chi_{2}$ & 0.012 & 0.011 & 0.010 & 0.012 & 0.010 & 0.010 & 0.012 \\
$\alpha_{1}$ & 5.0 & 5.0 & 5.0 & 5.0 & 5.0 & 5.0 & 5.0 \\
$\alpha_{2}$ & 10.0 & 10.0 & 10.0 & 10.0 & 10.0 & 10.0 & 10.0 \\
$\beta$ & 12.0 & 12.5 & 12.5 & 12.5 & 13.0 & 13.0 & 12.5 \\
$\zeta_{1}$ & 10000 & 10500 & 10500 & 10250 & 107500 & 10750 & 10250 \\
$\zeta_{2}$ & 50000 & 55000 & 55000 & 55000 & 60000 & 60000 & 55000 \\
\hline
\end{tabular}

It is assumed that all road users travel to one of the two CBDs during the morning and evening peak hours. The cost-flow relationship is specified as

$$
c(x, y)=0.0125+2.5 \times 10^{-6}\left(\sum_{n=1}^{2} \sum_{m=1}^{2}\left|\mathbf{f}_{m n}(x, y)\right|\right)^{1.3} \mathrm{~km} / \mathrm{h} .
$$

The instantaneous speed function is then

$$
|v(x, y)|=1 / c(x, y)=1 /\left(0.0125+2.5 \times 10^{-6}\left(\sum_{n=1}^{2} \sum_{m=1}^{2}\left|\mathbf{f}_{m n}(x, y)\right|\right)^{1.3}\right) \mathrm{km} / \mathrm{h} .
$$

The value of time $p_{m}$ for Class 1 and Class 2 commuters is $10 \mathrm{CNY} / \mathrm{h}$ and $20 \mathrm{CNY} / \mathrm{h}$, respectively. The perceived CBD cost function consists of a biased component and a demandrelated component. The biased component is assumed to be $\theta_{11}=12.0 \mathrm{CNY}, \theta_{12}=16.0 \mathrm{CNY}$, $\theta_{21}=40.0 \mathrm{CNY}$ and $\theta_{22}=30.0 \mathrm{CNY}$. The market externalities of the CBDs are defined as follows:

Class 1 road users travel to CBD 1: $S_{11}(x, y)=8.0 \times 10^{-11}\left(V_{1}-250000\right)^{2}$ CNY. 
Class 1 road users travel to CBD 2: $S_{12}(x, y)=4.0 \times 10^{-11}\left(V_{2}-200000\right)^{2} \mathrm{CNY}$.

Class 2 road users travel to CBD 1: $S_{21}(x, y)=16.0 \times 10^{-11}\left(V_{1}-250000\right)^{2} \mathrm{CNY}$.

Class 2 road users travel to CBD 2: $S_{22}(x, y)=8.0 \times 10^{-11}\left(V_{2}-200000\right)^{2} \mathrm{CNY}$.

Equation (25) assumes that the prevailing wind travels from the southeast to the northwest at a speed of $5.0 \mathrm{~m} / \mathrm{s}$. For easy computation, the coordinates are first transformed by rotating the modeling region by $135^{\circ}$ clockwise, so that the wind direction is from left to right (the same direction as the $x$ axis) during the computation process. The Pasquill stability category is $\mathrm{D}$, and thus $\sigma_{y}=0.16 x(1+0.0004 x)^{-1 / 2}, \sigma_{z}=0.14 x(1+0.0003 x)^{-1 / 2}$.

It is also assumed that the existing housing provision $H(x, y)$ depends on the housing's distance from the two CBDs, and that the maximum potential housing provision is $h_{\max }=250$ units $/ \mathrm{km}^{2}$. The closer the distance to a CBD, the less the residential housing provision. Variables $d_{1}, d_{2}$ are the distances to the CBDs, $d_{1}=\sqrt{(x-68.04)^{2}+(y-49.01)^{2}}$, and $d_{2}=\sqrt{(x-97.78)^{2}+(y-20.41)^{2}}$. It is then assumed that

$$
H(x, y)=h_{\max } \times\left(1-\exp \left(-\left(d_{1} / 5.0\right)^{1.5}\right)\right) \times\left(1-\exp \left(-\left(d_{2} / 5.0\right)^{1.5}\right)\right),
$$

where $(68.04,49.01)$ and $(97.78,20.41)$ are the centers of the two CBDs.

(a) Class 1 users traveling to CBD 1 (b) Class 1 users traveling to CBD 2
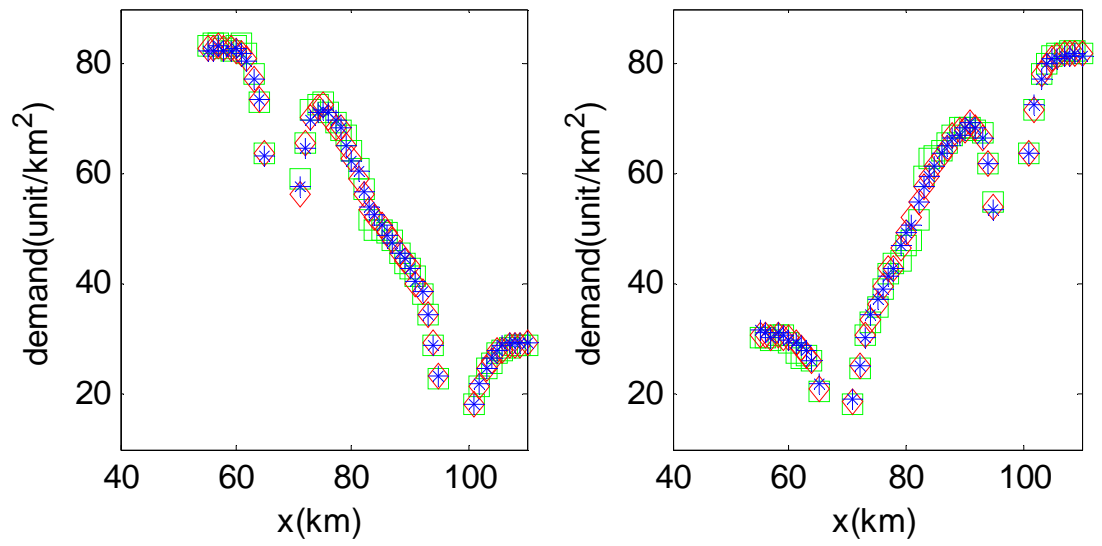

mesh1

mesh2

(c) Class 2 users traveling to CBD 1

(d) Class 2 users traveling to CBD 2
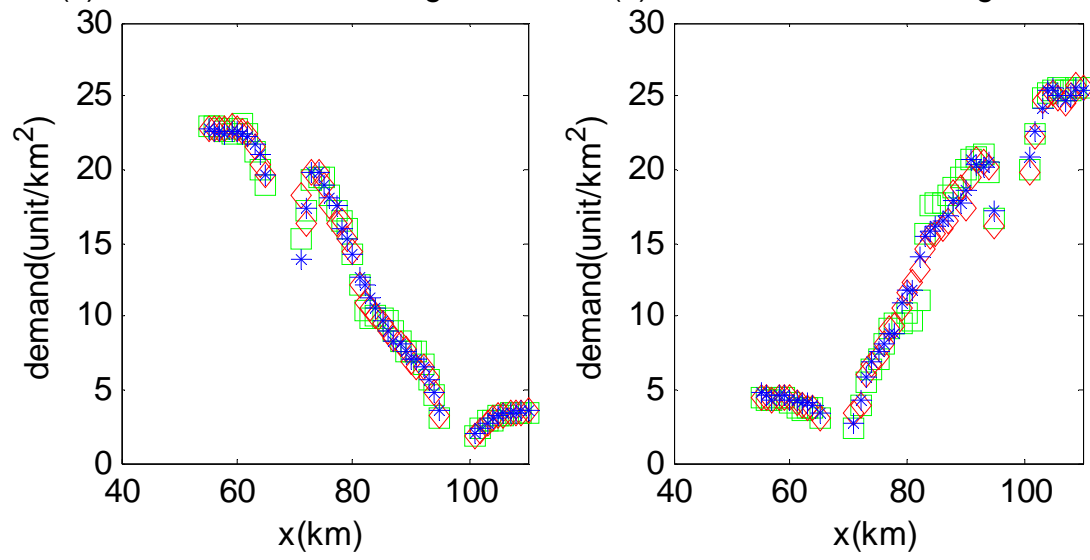

Figure 3: Convergence check 
The acceptable error is taken as $\varepsilon=10^{-5}$, and the smallest search interval for the golden section method is $\delta=0.02$. To check the convergence of this numerical method, three meshes of triangular elements are tested (mesh 1: 387 nodes and 664 elements, mesh 2: 457 nodes and 797 elements, mesh 3: 526 nodes and 925 elements). The demand curves along the line $y=-\frac{28.6}{29.74}(x-97.78)+20.41$ are plotted in Figure 3, and they indicate good convergent properties. Mesh 3 is adopted for the discussion that follows. The whole computation took about 16,151 seconds with the hpcpower2 system (http://www.hku.hk/cc/ccsystem/hpcpower2/), which was provided by the Computer Center of the University of Hong Kong.

Table 3 shows the degrees of attractiveness of the two CBDs and the way in which the total demand is split between them. The results show that the most important factor influencing the attractiveness of the CBDs is their locations. In this case, as the area adjacent to CBD 2 is much larger than that of CBD 1, more people will patronize CBD 2, including both Class 1 and Class 2 road users. Several other factors are also influential, such as the externalities of the CBDs. Both the biased parts of these externalities (assumed to be fixed) and the demandrelated parts (assumed to be sensitive to the volume of demand) may affect the demand of the CBDs, but these effects are comparatively non-obvious in this case.

Table 3: Splitting of the demand between CBDs (veh/h)

\begin{tabular}{cccc}
\hline$Q_{11}$ & $Q_{12}$ & $Q_{21}$ & $Q_{22}$ \\
\hline 148385 & 171615 & 32428 & 47572 \\
\hline
\end{tabular}

Figure 4 shows the travel cost for Class 1 road users. The travel cost for Class 2 road users is twice that of Class 1 users, as the travel cost increases with the distance to the destination. Furthermore, for road users who live near the CBDs, the travel cost contours for cases when the road users need to travel to the more distant CBD are not very regular. This irregularity results from the travel flow intensity, which is much higher because the road users cannot travel across the CBD directly, and this difficulty increases their travel cost. Figure 4 also shows that due to the comparatively higher flow intensity (shown in Figure 5c) and the associated lower travel speed, the travel cost increases faster in the area between the CBDs. 
(a) Class 1 users traveling to CBD 1

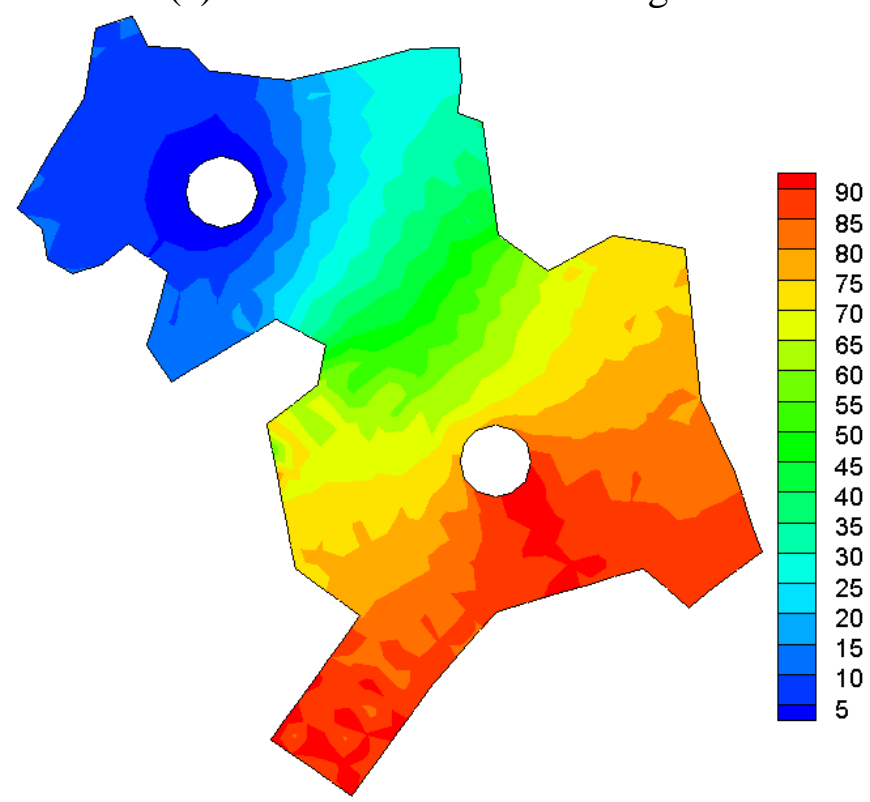

(b) Class 1 users traveling to CBD 2

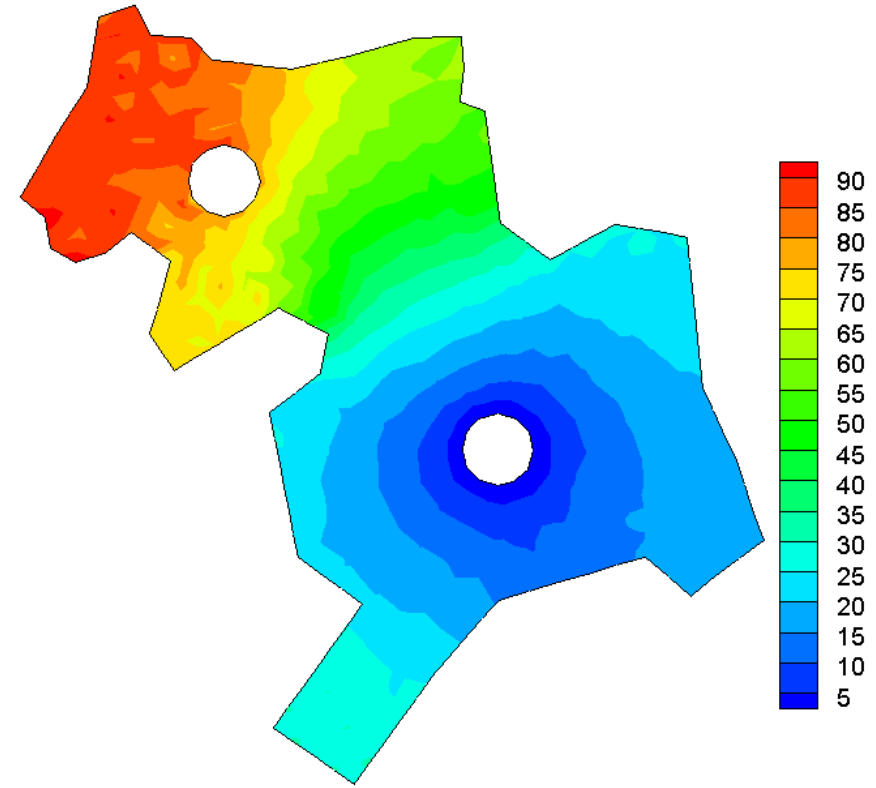

Figure 4: Travel cost for Class 1 road users (CNY)

Figure 5 shows the flow pattern of the travelers. Figure $5 c$ reveals that the flow intensity near regions $A$ and $B$ is relatively higher. This pattern occurs because the region between CBD 1 and CBD 2 is comparatively narrow (and acts as a bottleneck). At the same time, there is a considerable volume of demand for travel across this area. For many of those who live around CBD 2, the least expensive route for traveling to CBD 1 is near the boundary (regions $A$ and $B$ ), as shown in Figure 5 a (the relatively longer vector). The same pattern can be seen in Figure 5b, and for Class 2 road users the situation is similar. 
(a) Flow trajectories of Class 1 users traveling to CBD 1

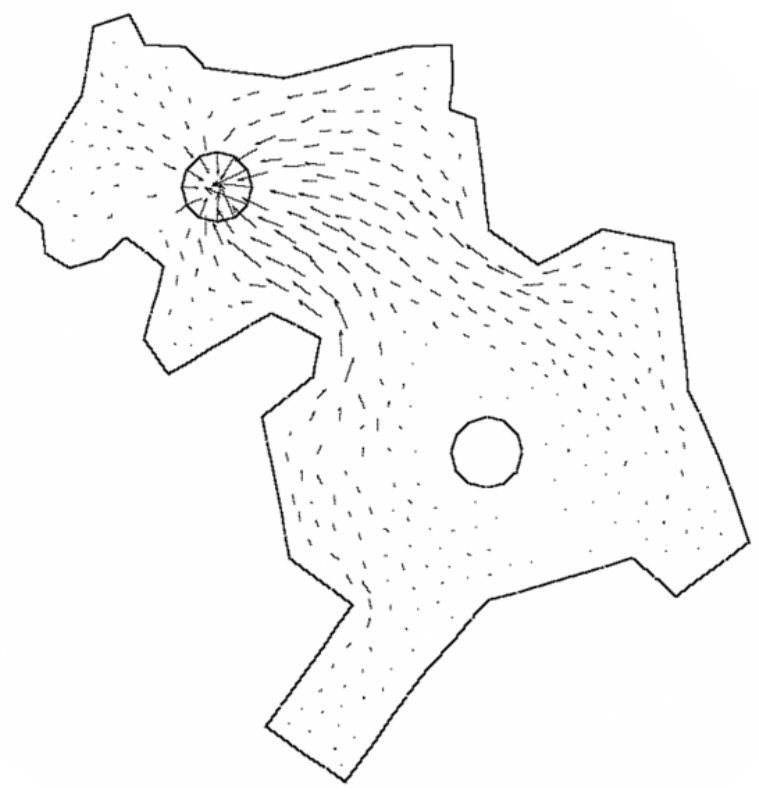

(b) Flow trajectories of Class 1 users traveling to CBD 2

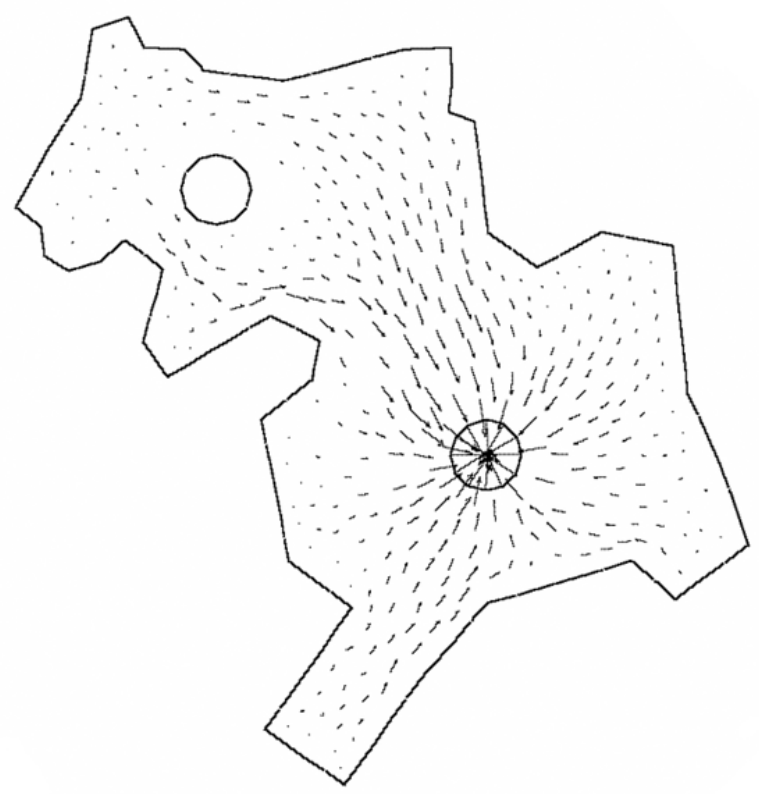


(c) Total flow intensity (veh/h/km)

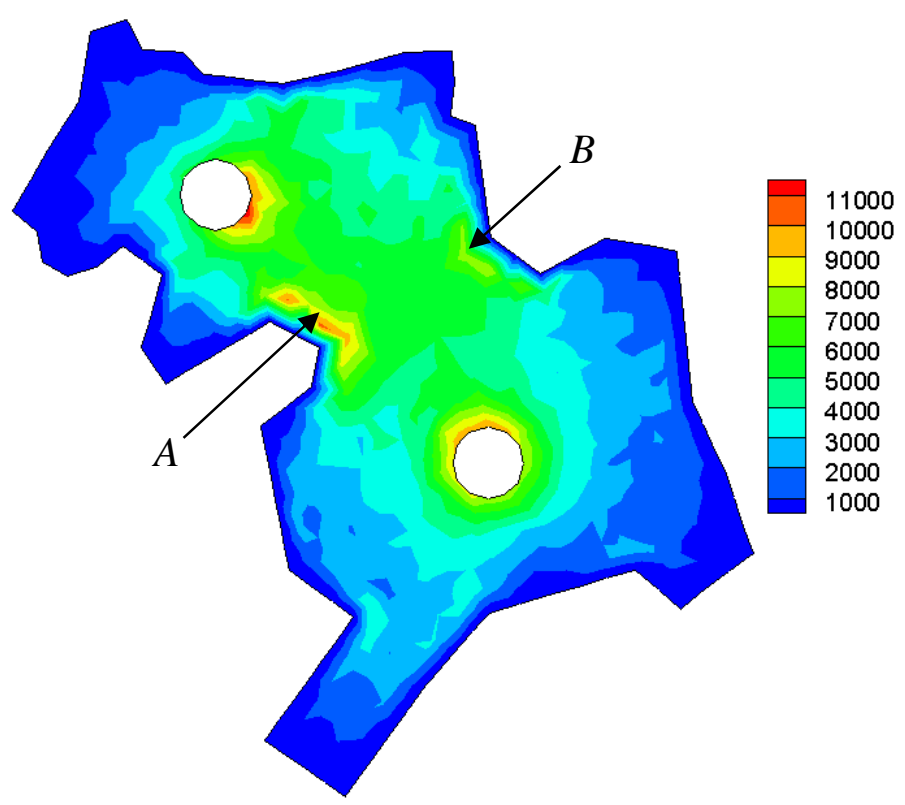

Figure 5: Flow pattern

Figure 6 shows the housing rent prices for Class 1 road users. As the provision-demand relationship is the same as for Class 2 road users, the two housing rent patterns are quite similar. The housing rent is extremely high near the CBDs, because the provision of housing in these places is lower, which is logical, as most of the buildings provided around the CBDs are for commercial activities. For most parts of the modeled region, the housing rent changes only slightly, and at a comparatively low level. Furthermore, in this case, the region $A$ (between the two CBDs), has a relatively lower housing rent due to the congestion and lower air quality to which this region is subject.

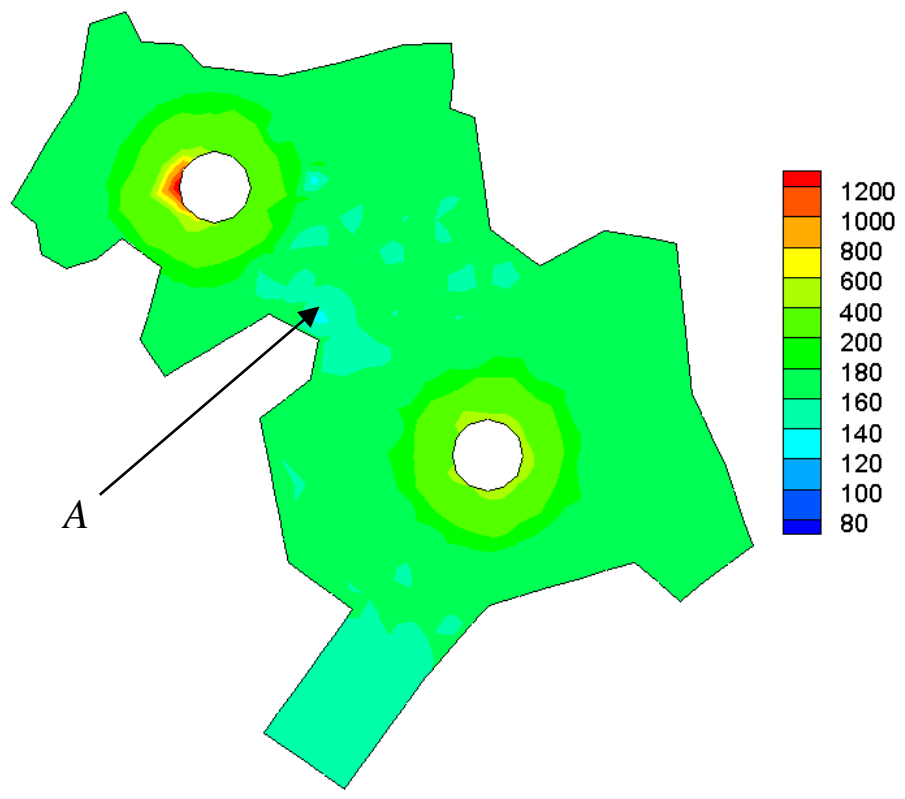

Figure 6: Housing rent for Class 1 road users (CNY) 
Figure 7 shows the pollutant concentration $\left(\mathrm{NO}_{x}\right)$ at ground level, $z=0$. Given the prevailing wind from the southeast, this figure clearly shows that the upwind locations are much less polluted than the downwind locations, due to the dispersion effect. At the same time, there is a positive relationship between traffic flow intensity and pollutant concentration. Therefore, the locations with higher traffic flow intensity have higher emissions, which results in a higher pollutant concentration. As the dispersion effect decreases sharply with distance, locations far from the emissions sources have a negligible effect from pollution. As it is assumed that there are no sources of emissions in the CBDs, the pollutant concentrations in the downwind locations are lower than those in the upwind locations.

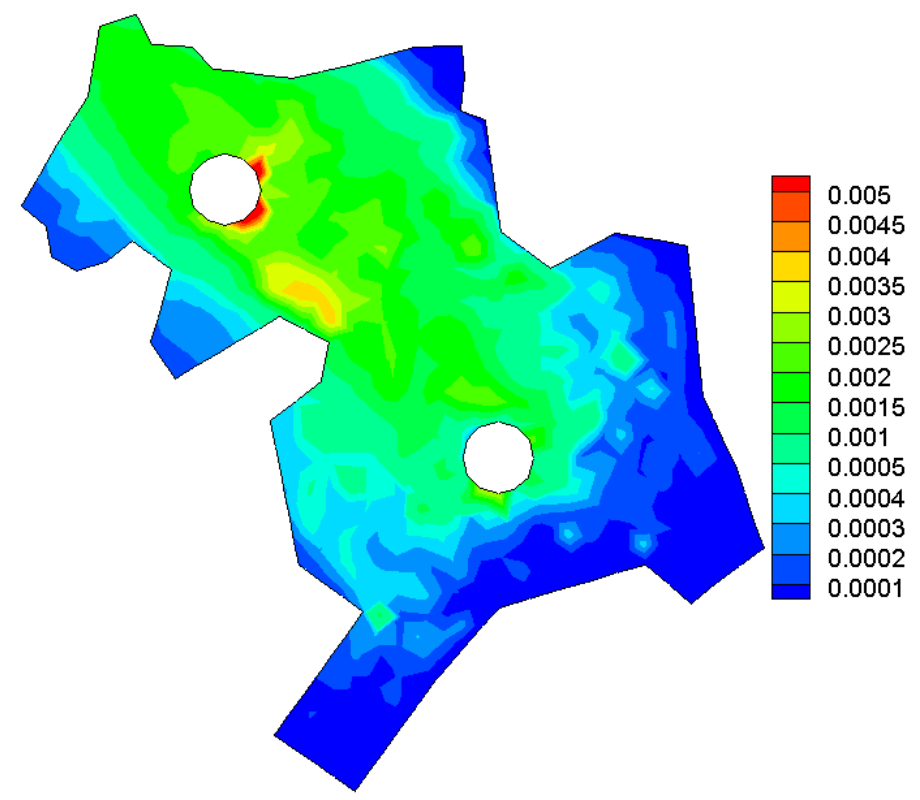

Figure 7: Pollutant concentrations $\left(\mathrm{mg} / \mathrm{m}^{3}\right)$

Figure 8 shows the demand contours for each type of road user and for the total demand distribution over the city. The figures in the left column represent the demand distribution without consideration of air quality, and the figures in the right column represent the demand distribution with air quality considered. Given the costs of travel, people prefer to live closer to the CBDs, but the housing rents closer to the CBDs are relatively higher, and the air quality there is lower. Hence, the actual demand distribution is a tradeoff among all of these factors. A comparison of the two columns in Figure 8, taking account of the prevailing wind (which is assumed to emanate from the southeast), shows that the distribution type for Class 1 road users is almost uniform, as these road users do not see air quality as an important factor when making housing location decisions. However, variation can be clearly seen in the behavior of Class 2 road users, as is particularly obvious in the regions adjacent to the commuters' destinations. As the air quality around the CBDs is poor, fewer people live there, and the downwind regions are affected more than the upwind regions. This shift can be also seen in Figures 8i and 8j. 
(a) Class 1 users traveling to CBD 1

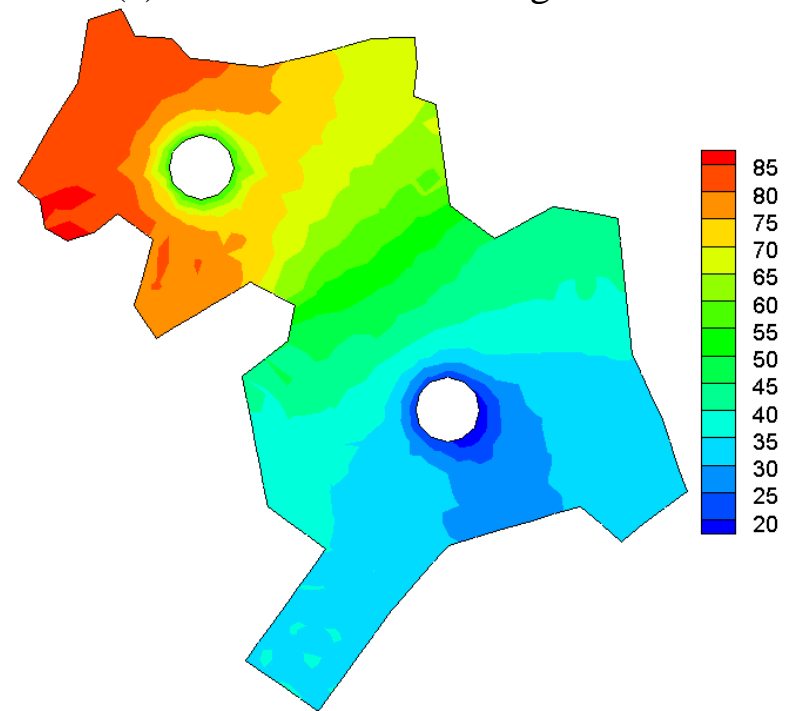

(c) Class 1 users traveling to CBD 2

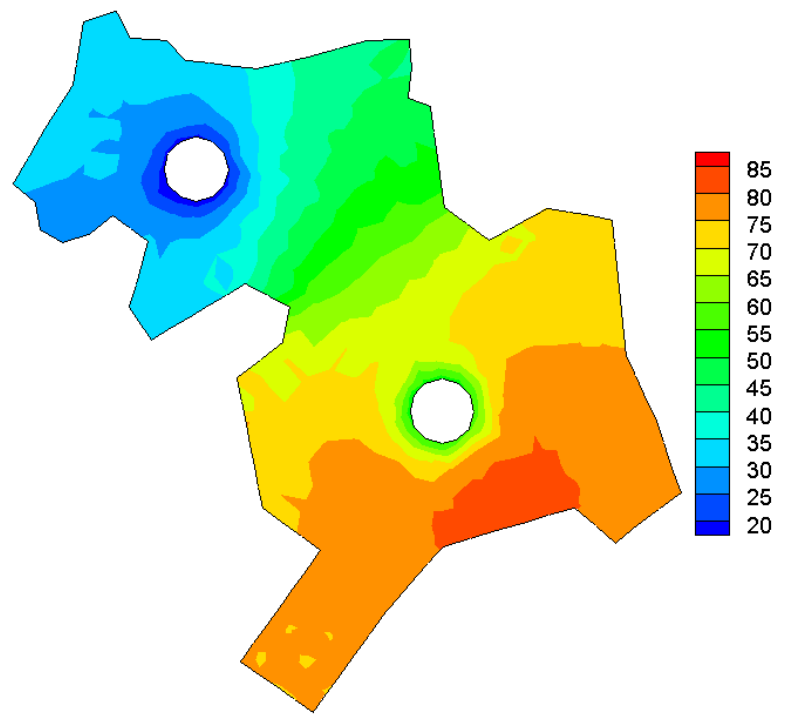

(e) Class 2 users traveling to CBD 1

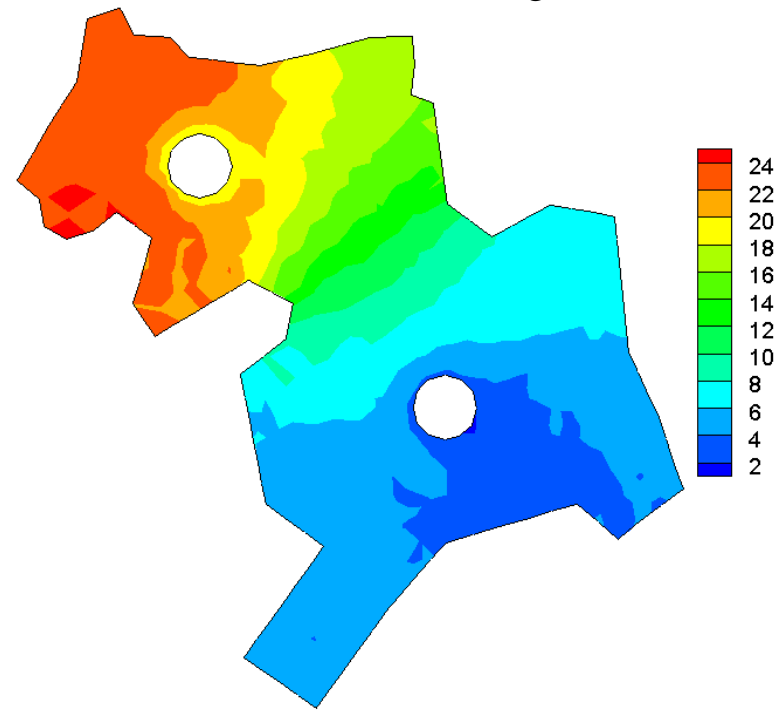

(b) Class 1 users traveling to CBD 1

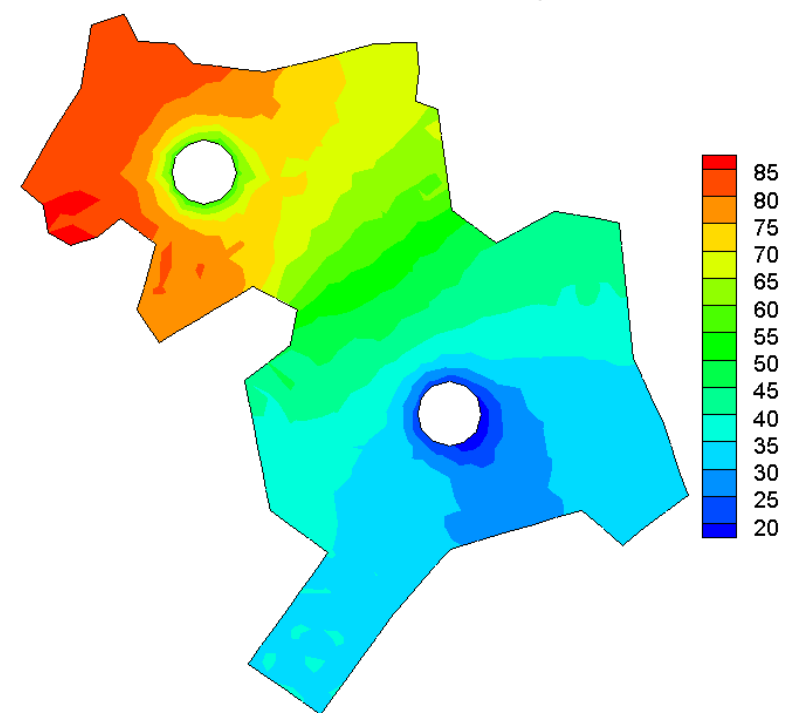

(d) Class 1 users traveling to CBD 2

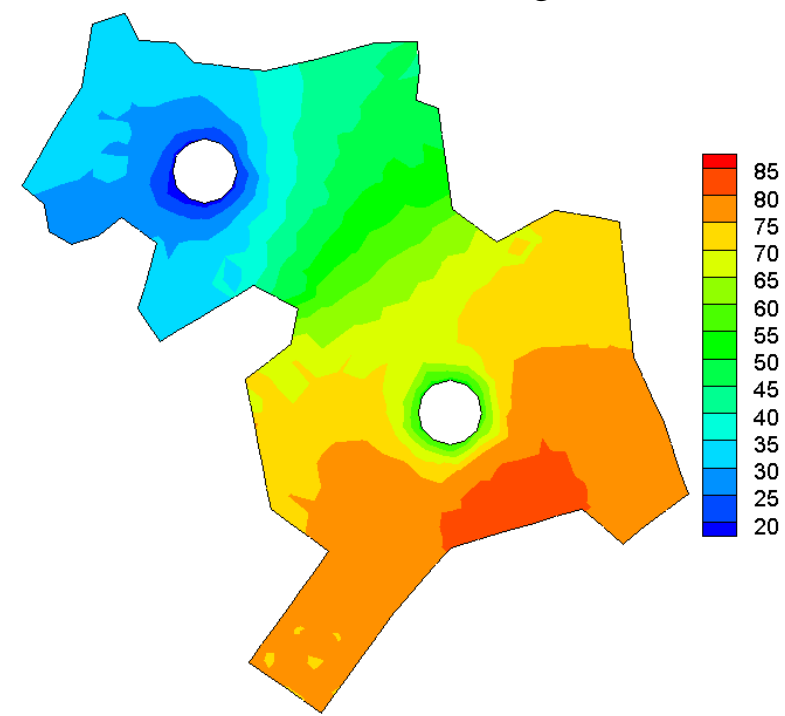

(f) Class 2 users traveling to CBD 1

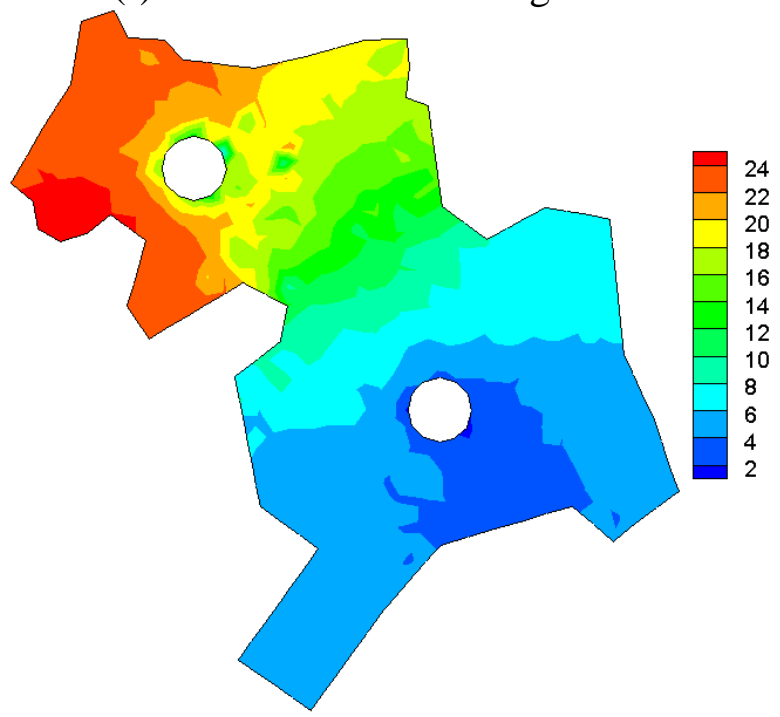


(g) Class 2 users traveling to CBD 2

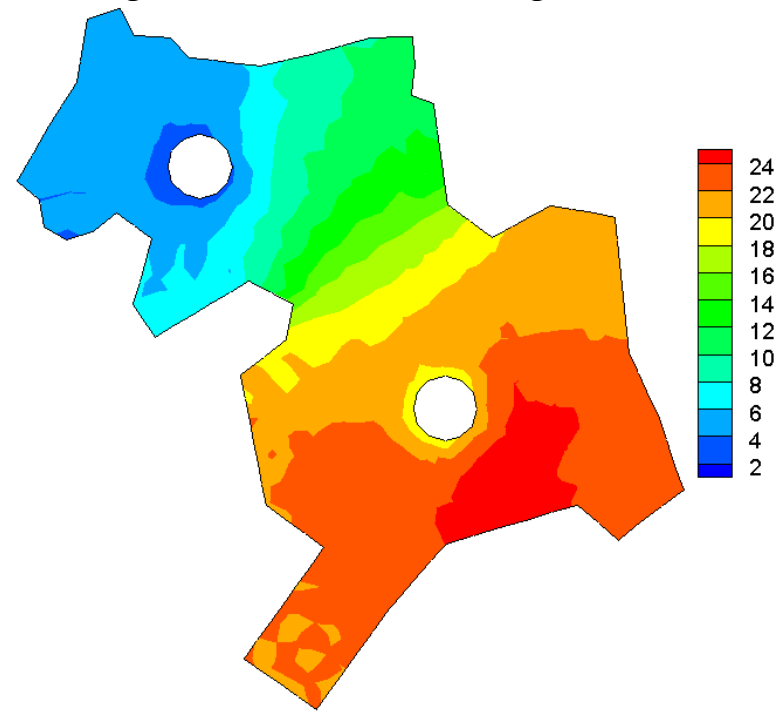

(i) Total demand from both types of road users

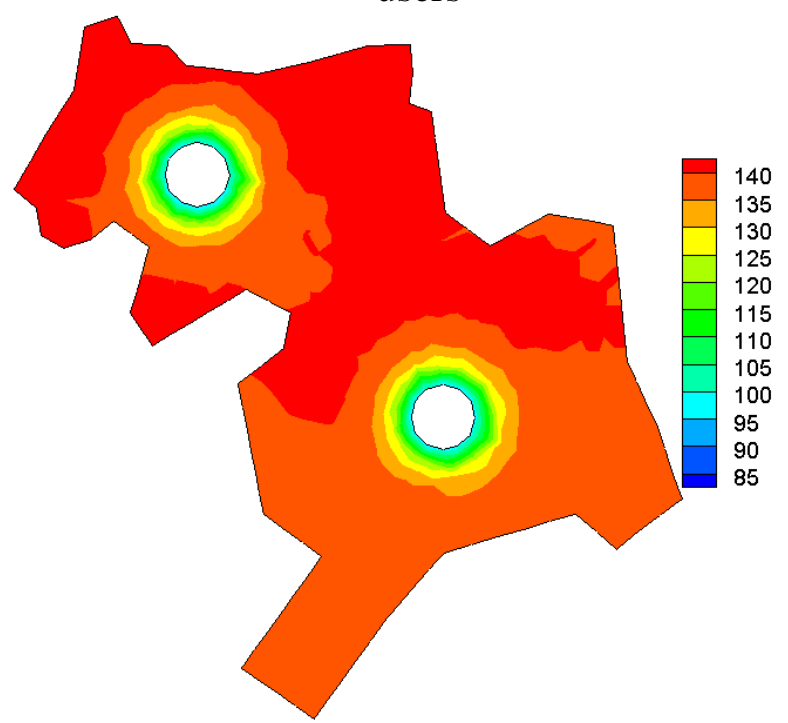

(h) Class 2 users traveling to CBD 2

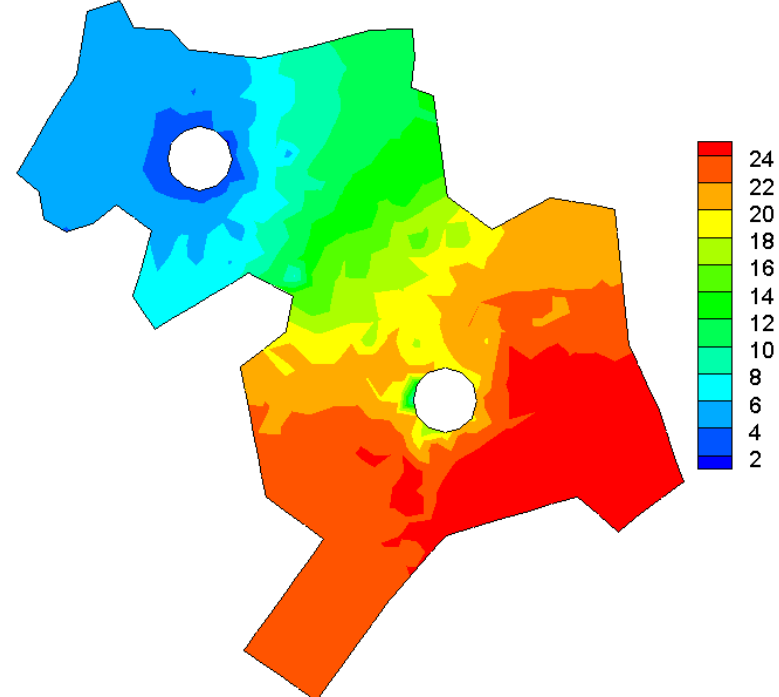

(j) Total demand from both types of road users

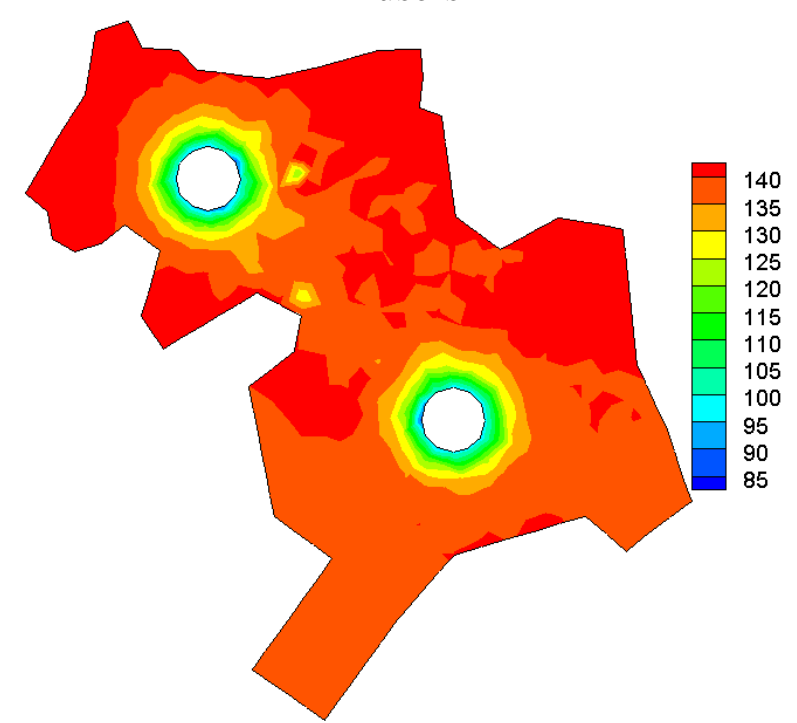

Figure 8: Demand contours: without the dispersion effect (left column, a, c, e, g and i) and with the dispersion effect (right column, b, d, f, h and j) (veh/h/km²)

\section{Conclusions}

In this study, the continuum modeling approach is adopted to study how air quality affects people's choices of residence location. The integrated land use, transport and environment model assumes that transportation is the only source of emissions. For each housing provision pattern there is a specific housing choice and traffic pattern, and in turn the related emissions affect people's housing location choices. The FEM is adopted to solve this problem numerically. There are several possible extensions to the modeling approach described in this study. As we use $\mathrm{NO}_{x}$ to represent the pollutants generated from transportation, we may use the Air Pollution Index instead in future studies. Although we model only the work trips taken during peak hours in this study, a dynamic continuum model which incorporates other trip purposes could also be developed in the future studies. 


\section{Acknowledgements}

The work described in this paper was supported by grants from the Research Grants Council of the Hong Kong Special Administrative Region, China (Project No.: 17208614), the National Research Foundation of Korea funded by the Korea government (MSIP) (NRF2010-0029446), and the National Natural Science Foundation of China (No. 71371143).

\section{References}

AHN, K., RAKHA, H., TRANI, A. \& VAN AERDE, M. 2002. Estimating vehicle fuel consumption and emissions based on instantaneous speed and acceleration levels. Journal of Transportation Engineering-Asce, 128, 182-190.

AHN, K., TRANI, A. A., RAKHA, H. \& VAN AERDE, M. 1999. Microscopic fuel consumption and emission models. Transportation Research Board 78th Annual Meeting. Washington, D. C.

BEN-AKIVA, M. \& BOWMAN, J. L. 1998. Integration of an activity-based model system and a residential location model. Urban Studies, 35, 1131-1153.

BEN-AKIVA, M., LITINAS, N. \& TSUNOKAWA, K. 1985. Continuous spatial choice: The continuous logit model and distributions of trips and urban densities. Transportation Research Part A: General, 19, 119-154.

BEN-AKIVA, M. \& WANTANTADA, T. 1981. Application of a continuous spatial choice logit model. In: MANSKI, C. F. \& MCFADDEN, D. (eds.) Structural Analysis of Discrete Choice Data with Econometric Applications. Cambridge, MA: MIT Press.

BHAT, C. R. \& GUO, J. 2004. A mixed spatially correlated logit model: Formulation and application to residential choice modeling. Transportation Research Part B: Methodological, 38, 147-168.

DU, Y. C., WONG, S. C. \& SUN, L. J. 2016. A multi-commodity discrete/continuum model for a traffic equilibrium system. Transportmetrica A: Transport Science, 12, 249-271.

ELLICKSON, B. 1981. An alternative test of the hedonic theory of housing markets. Journal of Urban Economics, 9, 56-79.

EPA, 2010. Dispersion Modeling [Online]. Available: http:/www.epa.gov/scram001/dispersionindex.htm [Assessed 4-25-2011]

EPA, 2011. Preferred/Recommended Models [Online]. Available: www.epa.gov/ttn/scram/dispersion_prefrec.htm\#calpuff [Assessed 4-25-2011]

GIULIANO, G. 1989. Research policy and review 27. New directions for understanding transportation and land use. Environment and Planning A, 21, 145-159.

HO, H. W. \& WONG, S. C. 2005. Combined model of housing location and traffic equilibrium problems in a continuous transportation system. The 16th International Symposium on Transportation and Traffic Theory (ISTTT16). Maryland, U.S.A.

HO, H. W. \& WONG, S. C. 2006. Two-dimensional continuum modeling approach to transportation problems. Journal of Transportation Systems Engineering and Information Technology, 6, 53-68.

HO, H. W. \& WONG, S. C. 2007. Housing allocation problem in a continuum transportation system. Transportmetrica, 3, 21-39.

HUH, S. \& KWAK, S. J. 1997. The choice of functional form and variables in the hedonic price model in Seoul. Urban Studies, 34, 989-998.

ORFORD, S. 2000. Modeling spatial structures in local housing market dynamics: A multilevel perspective. Urban Studies, 37, 1643-1671. 
ROSEN, S. 1974. Hedonic prices and implicit markets: Product differentiation in pure competition. Journal of Political Economy, 82, 34-55.

VAUGHAN, R. 1987. Urban Spatial Traffic Patterns, London, Pion.

WAGNER, P. \& WEGENER, M. 2007. Urban land use, transport and environment models. Experiences with a integrated microscopic approach. disP, 170, 45-56.

WARDMAN, M. \& BRISTOW, A. L. 2004. Traffic related noise and air quality valuations: Evidence from stated preference residential choice models. Transportation Research Part D: Transport and Environment, 9, 1-27.

WEGENER, M. 2004. Overview of land-use transport models. In: HENSHER, D. A. \& BUTTON, K. (eds.) Transport Geography and Spatial Systems: Handbook 5 of the Handbook in Transport. Kidlington, UK: Pergamon/Elisevier Science.

WEGENER, M. \& FUERST, F. 2004. Land-use Trasnport Interaction: State of the Art. Institut für Raumplanung, Fakultät Raumplanung, Universität Dortmund, Dortmund, Germany.

WHEATON, W. C. 1977. Income and urban residence: An analysis of consumer demand for location. The American Economic Review, 67, 620-631.

WONG, S. C. 1998. Multi-commodity traffic assignment by continuum approximation of network flow with variable demand. Transportation Research Part B: Methodological, 32, 567-581.

WONG, S. C., LEE, C. K. \& TONG, C. O. 1998. Finite element solution for the continuum traffic equilibrium problems. International Journal for Numerical Methods in Engineering, 43, 1253-1273.

WONG, W. \& WONG, S. C. 2015. Systematic bias in transport model calibration arising from the variability of linear data projection. Transportation Research Part B, 75, 118.

WONG, W. \& WONG, S. C. 2016. Network topological effects on the macroscopic Bureau of Public Roads function. Transportmetrica A: Transport Science, 12, 272-296.

ZONGDAG, B. \& PIETERS, M. 2005. Influence of accessibility on residential location choice. Transportation Research Record: Journal of the Transportation Research Board, 1992, 63-70. 